\title{
Cermets Ni-GDC para su uso como ánodos en IT-SOFC basadas en electrolitos GDC
}

\author{
V. GIL, J.TARTAJ, C. MOURE \\ Instituto de Cerámica y Vidrio, CSIC. Campus de Cantoblanco, Madrid, España
}

\begin{abstract}
El objetivo de este trabajo es estudiar en un amplio rango de temperaturas $\left(1000-1450^{\circ} \mathrm{C}\right)$ y mediante difracción de rayos- $\mathrm{X}$ (DRX) las posibles reacciones en estado sólido que pueden tener lugar en las regiones interfaciales de sistemas constituidos por composites $\mathrm{NiO}-\mathrm{Ce}_{09} \mathrm{Gd}_{01} \mathrm{O}_{195}\left(\mathrm{NiO}-\mathrm{GDC}\right.$ ) con un $50 \%$ en peso de $\mathrm{NiO}$, y de Cermets Ni- $\mathrm{Ce}_{0.9} \mathrm{Gd}_{01} \mathrm{O}_{195}$ (Ni-GDC) con un $39 \%$ en volumen $\mathrm{Ni}$, y electrolitos basados en soluciones sólidas $\mathrm{Ce}_{0.9} \mathrm{Gd}_{01} \mathrm{O}_{195}$ (GDC). Y así mismo, estudiar la compatibilidad termoquímica y termomecánica entre los distintos materiales cerámicos que constituyen los sistemas electrolito/ánodo. Se confirma mediante MEB y análisis EDAX que los sistemas cosinterizados entre 1350 y $1400^{\circ} \mathrm{C}$ durante $2 \mathrm{~h}$ y constituidos por un electrolito basado en ceria (GDC) y un ánodo basado en un cermet níquel-ceria (Ni-GDC), presentan una buena adherencia entre capas, sin la formación de defectos y sin la presencia de interdifusión de especies a lo largo de la región interfacial. Es decir, se confirma que ambos materiales, Ni-GDC y GDC son compatibles termoquímica y termomecánicamente.
\end{abstract}

Palabras clave: $\mathrm{CeO}_{2}-\mathrm{Gd}_{2} \mathrm{O}_{3}$, Ni-CGO cermet, compatibilidad química, compatibilidad termomecánica.

\section{Ni-GDC anode for GDC electrolyte-based IT-SOFC}

The purpose of this work is to investigate the possible solid state reactions between the $\mathrm{NiO}-\mathrm{Ce}_{0.9} \mathrm{Gd}_{0.1} \mathrm{O}_{1.95}(\mathrm{NiO}-\mathrm{GDC})$ composites with $50 \%$ wt $\mathrm{NiO}$, and cermets $\mathrm{Ni}-\mathrm{Ce}_{09} \mathrm{Gd}_{01} \mathrm{O}_{195}$ (Ni-GDC) with 39 vol. \% of Ni, and electrolyte solid solutions based on $\mathrm{Ce}_{0.9} \mathrm{Gd}_{0.1} \mathrm{O}_{1.95}$ (GDC) employing the X-ray diffraction technique. At the same time the thermochemical and thermomechanical compatibility between these materials was established.

Results obtained from SEM and EDAX analysis showed that the electrolyte based on ceria (GDC) and the anode based on nickel-ceria cermet (Ni-GDC) present good adhesion and well defined interfaces. No reactive phases and other type of defects were detected. This study showed that both materials, Ni-GDC and GDC are thermochemical and thermomechanically compatible.

Key words: $\mathrm{CeO}_{2}-\mathrm{Gd}_{2} \mathrm{O}_{3}, \mathrm{Ni}-\mathrm{CGO}$ cermet, thermal compatibility, chemical compatibility.

\section{INTRODUCCIÓN}

Debido a su excelente capacidad catalítica en la oxidación del combustible y a su alta conductividad electrónica además de bajo coste en comparación con otros metales, el níquel metálico ha sido seleccionado ampliamente como un buen material anódico para pilas de combustible de óxido sólido (SOFC) (1-2). Sin embargo, al ser un metal presenta unos valores de coeficientes de expansión térmica muy diferentes a los de los electrolitos normalmente utilizados, lo que implica un mal acoplamiento termomecánico y en consecuencia el mal funcionamiento de la pila. En este sentido, la incorporación de un material cerámico al ánodo, resolvería este problema. Así pues, el material más utilizado como ánodo es un cermet poroso de metal y cerámica, en concreto Ni/YSZ. Mientras que la introducción del metal en la matriz cerámica permite obtener una conductividad electrónica suficiente así como un alto rendimiento (3), el papel principal de la cerámica en el cermet, además de aportar la conductividad iónica necesaria, es de carácter estructural, constituyendo una red tridimensional sobre la que se dispersan las partículas metálicas, en este caso de níquel. Además, actúa como inhibidor de la agregación y crecimiento de grano de las partículas de níquel, tanto durante la fabricación de los componentes como en la operación e inhibe la coalescencia de éstas durante la consolidación y funcionamiento de la pila.

Por otra parte, en los últimos tiempos se tiende hacia la búsqueda de nuevos conductores iónicos que mejoren la conductividad del electrolito y permitan así disminuir la temperatura de trabajo de la SOFC $\left(600-800^{\circ} \mathrm{C}\right)$. Dentro de estos materiales los mejores candidatos como electrolitos son los basados en ceria dopada, y más concretamente los dopados con gadolinia, de composición $\mathrm{Ce}_{0.9} \mathrm{Gd}_{0.1} \mathrm{O}_{1.95}$ (4).

En el presente trabajo se aborda el estudio de la compatibilidad química y termomecánica entre un electrolito $\mathrm{Ce}_{0.9} \mathrm{Gd}_{0.1} \mathrm{O}_{1.95}$ (GDC) y un cermet constituido por níquel (material metálico) y el mismo material cerámico que el electrolito, $\mathrm{Ce}_{0.9} \mathrm{Gd}_{0.1} \mathrm{O}_{1.95}(\mathrm{Ni} / \mathrm{GDC})$, con el fin de ajustar lo máximo posible los coeficientes de expansión térmica entre el electrolito y el ánodo. 


\section{PARTE EXPERIMENTAL}

Los correspondientes cermets de $\mathrm{Ni}-\mathrm{Ce}_{0.9} \mathrm{Gd}_{0.1} \mathrm{O}_{1.95}(39 \%$ vol. Ni) se han obtenido a partir de la síntesis de polvos de composites $\mathrm{NiO}-\mathrm{Ce}_{0.9} \mathrm{Gd}_{0.1} \mathrm{O}_{1.95}(50 \%$ en peso $\mathrm{NiO})$ tras posterior reducción de las matrices cerámicas preparadas a partir de ellos. El método de síntesis seleccionado (PCS-EG) se basa en la preparación de una solución acuosa de complejos, que contienen los cationes de interés, y son polimerizables con etilenglicol, de este modo, a través de la eliminación de agua se forma un gel que es posteriormente pirolizado (5). Dichos polvos han sido sometidos a un tratamiento de calcinación a $800^{\circ} \mathrm{C}$ durante $1 \mathrm{~h}$, proceso tras el cual se obtienen unos polvos con dos fases cristalinas bien diferenciadas, $\mathrm{NiO}$ y $\mathrm{Ce}_{0.9} \mathrm{Gd}_{0.1} \mathrm{O}_{1.95^{\prime}}$ y que serán nombrados en adelante como NiO-GDC.

En el caso de las cerámicas basadas en ceria, éstas fueron preparadas por dos vías alternativas: a partir de polvos nanoparticulados $\mathrm{Ce}_{0.9} \mathrm{Gd}_{0.1} \mathrm{O}_{1.95}$ sintetizados mediante una ruta de procesamiento química (precipitación de hidróxidos) (6) y a partir de un polvo comercial submicrónico de composición $\mathrm{Ce}_{0.9} \mathrm{Gd}_{0.1} \mathrm{O}_{1.95^{\prime}}$ el cual ha sido modificado con $1.0 \%$ peso de $\mathrm{Bi}_{2} \mathrm{O}_{3}$ para mejorar así el proceso de densificación vía fase líquida (7). Dichos polvos serán nombrados en adelante como GDC y GDC-Bi, respectivamente.

Para llevar a cabo los estudios de reactividad de los pares $\mathrm{NiO}-\mathrm{GDC}+\mathrm{GDC}$ y $\mathrm{NiO}-\mathrm{GDC}+\mathrm{GDC}-\mathrm{Bi}$ se mezclaron y homogeneizaron polvos al $50 \%$ en peso de cada uno de los componentes para posteriormente conformar discos por prensado isostático. Dichos discos fueron tratados térmicamente en aire entre 1350 y $1450^{\circ} \mathrm{C}$ durante $4 \mathrm{~h}$ y posteriormente fueron recocidos en aire a $1000^{\circ} \mathrm{C}$ durante $100 \mathrm{~h}$. El rango de temperaturas bajo estudio se seleccionó en función de los resultados de densificación previamente obtenidos para ambos tipos de electrolitos $(6,8)$. Tras los tratamientos térmicos, los discos fueron molidos y los polvos así obtenidos analizados mediante difracción de rayos-X (XRD) en un difractómetro Siemens D-5000 (radiación CuK $\alpha$, Alemania).

Para comprobar la adherencia entre las capas del ánodo y el electrolito, así como la formación de la interfase se prepararon sand wiches NiO-GDC/GDC y NiO-GDC/ GDC-Bi utilizando las mismas condiciones de prensado isostático que anteriormente. Una vez conformados los pares, éstos fueron sinterizados durante $2 \mathrm{~h}$ a 1400 y $1350^{\circ} \mathrm{C}$, respectivamente, y posteriormente reducidos a $700^{\circ} \mathrm{C}$ durante $2 \mathrm{~h}$ en atmósfera $\mathrm{N}_{2}$ $\mathrm{H}_{2}$ 90-10\% (5), con el objeto de disponer de electrolitos densos y de una alta conductividad iónica, ánodos porosos y altamente conductores electrónicos y además de una interfase ánodo/ electrolito bien definida. La microestructura de las secciones transversales de los pares (superficies pulidas y fracturas) fueron caracterizadas mediante microscopía electrónica de barrido (MEB) (Zeiss DSM 950, Oberkochem, Germany) y la posible interdifusión de ciertas especies a lo largo de la región interfacial fue observada vía análisis por energías dispersivas de rayos- $X$ (EDAX).

Los estudios de compatibilidad termomecánica entre los distintos materiales que componen los sistemas ánodo/ electrolito se llevaron a cabo mediante el cálculo de los coeficientes de expansión térmica (CET). Dichos coeficientes fueron obtenidos a partir de curvas de enfriamiento dilatométricas realizadas en aire y a una velocidad de calentamiento y enfriamiento de $5^{\circ} \mathrm{C} / \mathrm{min}$, utilizando un dilatómetro Netzsch modelo 407/E (Bayern, Alemania). Las propiedades eléctricas de los materiales que constituyen los pares fueron obtenidas mediante un analizador de impedancias HP-4192, tal y como se reporta en los trabajos elaborados por V.Gil y col. $(5,7)$.

\section{RESULTADOS Y DISCUSIÓN}

En la figura 1 se representan los difractogramas de rayos- $X$ correspondientes a mezclas al $50 \%$ en peso de polvos $\mathrm{NiO}-$ $\mathrm{Ce}_{0.9} \mathrm{Gd}_{0.1} \mathrm{O}_{1.95}$ (composite $\left.\mathrm{NiO}-\mathrm{GDC}\right)+\mathrm{Ce}_{0.9} \mathrm{Gd}_{0.1} \mathrm{O}_{1.95}$ (electrolito obtenido por vía química), tratados térmicamente entre 1350 y $1450^{\circ} \mathrm{C}$ durante $4 \mathrm{~h}$ y posteriormente recocidos en aire y durante un largo periodo de tiempo a la temperatura más común de funcionamiento, $1000^{\circ} \mathrm{C}$ durante $100 \mathrm{~h}$. Además, se representan los difractogramas iniciales "en verde" de cada uno de los polvos cerámicos empleados, $\mathrm{NiO}$ y GDC es decir, los difractogramas correspondientes a los polvos cerámicos antes de ser sometidos a tratamiento térmico. En todo el rango de temperaturas y tiempos es posible observar que los picos de difracción existentes y correspondientes a dos fases cristalinas bien diferenciadas, $\mathrm{NiO}$ y $\mathrm{Ce}_{0.9} \mathrm{Gd}_{0.1} \mathrm{O}_{1.95^{\prime}}$ no sufren ninguna transformación. Además, tampoco se detecta la presencia de nuevos picos de difracción. Por lo tanto, se puede afirmar que los polvos cerámicos de partida permanecen inalterados, sin degradarse ni reaccionar al menos hasta una temperatura de $1450^{\circ} \mathrm{C}$. Resultados similares fueron encontrados para el sistema en el que el electrolito fue modificado con una pequeña cantidad de $\mathrm{Bi}_{2} \mathrm{O}_{3}$ (NiO-GDC/GDC-Bi).

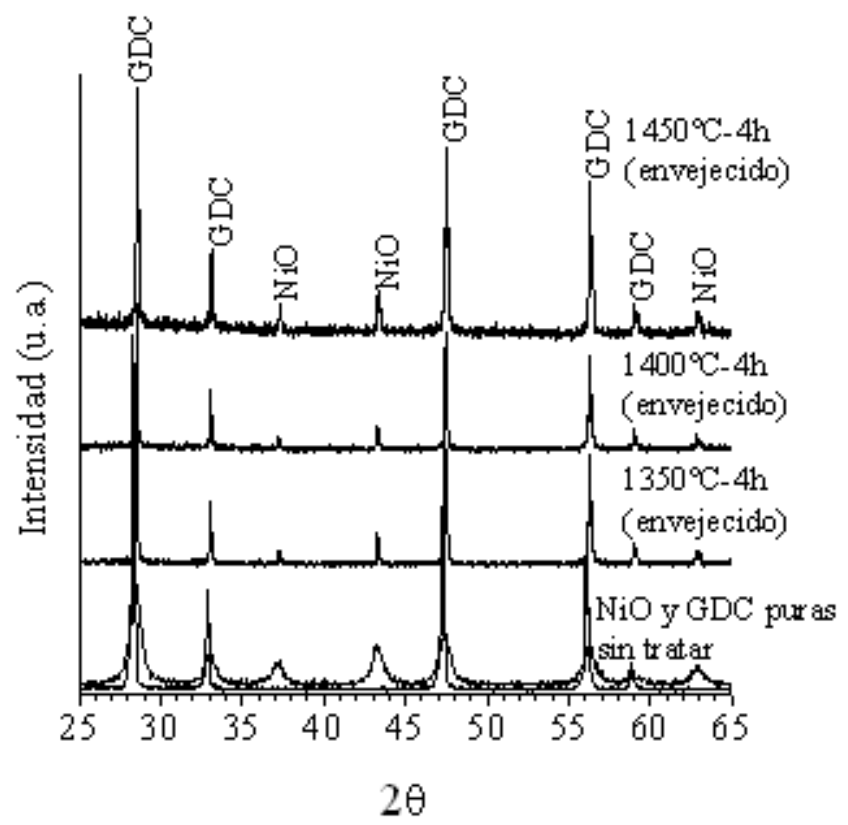

Fig. 1- Difractogramas de rayos- $\mathrm{X}$ de mezclas de polvos $\mathrm{NiO}-\mathrm{GDC}+$ GDC, sin tratar y tratadas térmicamente a diferentes temperaturas y posteriormente envejecidas en aire a $1000^{\circ} \mathrm{C}$ durante $100 \mathrm{~h}$.

Para comprobar la adherencia entre las capas de los pares ánodo/electrolito, los pares cosinterizados fueron caracterizados mediante microscopía electrónica de barrido (MEB). En principio, el estudio plantea en qué manera el proceso de reducción del composite para obtener el cermet 
puede afectar a las características microestructurales de las cerámicas empleadas. En la figura 2 se ilustran a modo de ejemplo, dos micrografías obtenidas por MEB de superficies pulidas de un sistema NiO-GDC/GDC-Bi antes y después de ser sometido al proceso de reducción $\left(700^{\circ} \mathrm{C}-2 \mathrm{~h}\right.$, en atmósfera $\mathrm{N}_{2}-\mathrm{H}_{2} 90-10 \%$ ). Se confirma que con dicho proceso únicamente sufre un cambio microestructural significativo el material cerámico correspondiente al ánodo, el cual aumenta su porosidad como consecuencia de la evolución de $\mathrm{O}_{2}$ gaseoso procedente de la transformación de $\mathrm{NiO}$ a $\mathrm{Ni}$ metálico. De acuerdo a los resultados obtenidos anteriormente, la caracterización microestructural de los pares se realizó en todos los casos sobre pares reducidos.
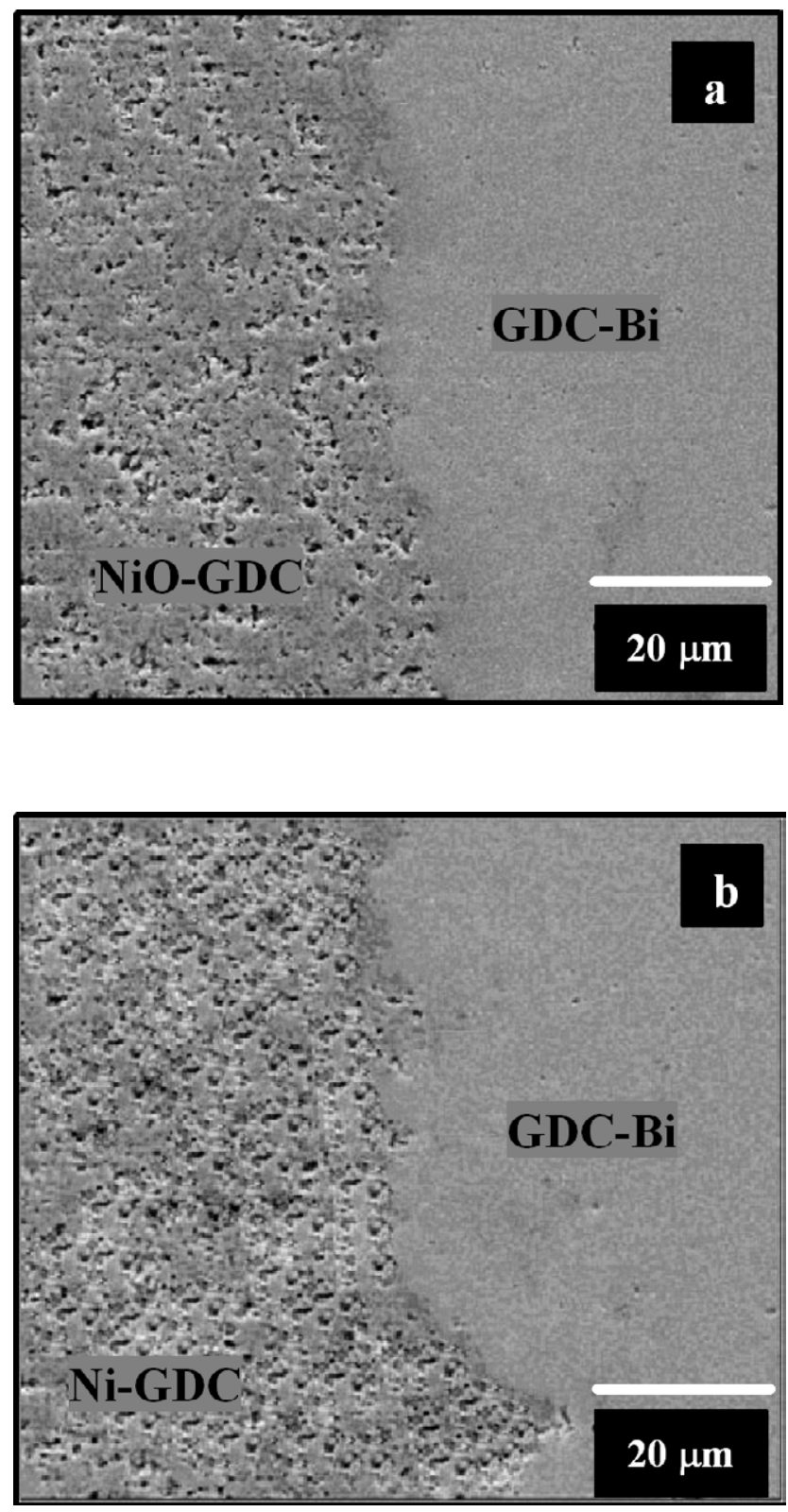

Fig. 2- Micrografías MEB de secciones transversales pulidas de sistemas ánodo/electrolito sinterizados a $1350^{\circ} \mathrm{C}$ durante $2 \mathrm{~h}$ antes y después de un proceso de reducción, (a) composite NiO-GDC/GDC-Bi y (b) cermet Ni-GDC/GDC-Bi.
En la figura 3 se muestran las secciones transversales (fracturas y superficies pulidas) obtenidas por MEB correspondientes a sistemas cermet/electrolito cosinterizados entre 1350 y $1400^{\circ} \mathrm{C}$ durante $2 \mathrm{~h}$. En todos los casos las interfases están perfectamente definidas, se mantiene la buena adherencia entre las capas del cermet y el electrolito y no se detectan a priori grietas o delaminaciones de las mismas. Este último aspecto sugiere que ambos materiales, $\mathrm{NiO}$ y GDC son compatibles termomecánicamente tal y como confirmaremos posteriormente.

Finalmente la figura 4 ilustra, a modo de ejemplo, los análisis por energías dispersivas de rayos-X (EDAX) realizados sobre zonas puntuales elegidas a lo largo de las regiones interfaciales del sistema Ni-GDC/GDC cosinterizado a $1400^{\circ} \mathrm{C}$. A partir de dichas gráficas se observa que en principio la región correspondiente al electrolito y próxima a la interfase (punto 2) no presenta picos característicos de la fase níquel y además, su análisis composicional es similar a una zona localizada en el electrolito y lejana de la zona común (punto 1). Por el contrario, una región localizada en el ánodo y cercana a la región interfacial (punto 3) presenta una composición de fases de Ni y ceria-gadolinia similar a la del interior del mismo (punto 4) y acorde con la composición del cermet inicialmente preparado, es decir, un $44 \%$ en peso de $\mathrm{Ni}$ (obtenido a partir de un composite con un $50 \%$ en peso de $\mathrm{NiO}$ ). Resultados similares fueron obtenidos en el caso del sistema Ni-GDC/GDC-Bi cosinterizado a menor temperatura $\left(1350^{\circ} \mathrm{C}\right.$ durante $\left.2 \mathrm{~h}\right)$. Estos resultados indican que a las temperaturas de sinterización estudiadas no existe ninguna difusión significativa de algún catión desde o hacia el electrolito, y por tanto no se aprecia la existencia de nuevas fases producidas por una posible reacción entre el ánodo y el electrolito, tal y como se observó en los análisis de difracción de rayos-X (figura 1).

Con el objeto de confirmar el correcto ajuste de los coeficientes de expansión térmica (CET) durante la cosinterización del conjunto en verde, se han calculado éstos a partir de las curvas dilatométricas de enfriamiento. En la Tabla I se observa cómo ambos electrolitos presentan unos CET similares entre sí $\left(\alpha_{\mathrm{GDC}}=\sim 12.6 \times 10^{-6} \mathrm{~K}^{-1}, \alpha_{\mathrm{GDC}-\mathrm{Bi}}=\sim 12.7 \times 10^{-6} \mathrm{~K}^{-1}\right)$ y coherentes con los reportados en la literatura para sistemas similares (10-11) $\left(\alpha=12.2-12.8 \times 10^{-6} \mathrm{~K}^{-1}\right)$. Respecto al ánodo, tal y como era de esperar, éste presenta un coeficiente de expansión térmica ( $\alpha$ $=\sim 13.5 \times 10^{-6} \mathrm{~K}^{-1}$ ) no muy elevado si es comparado con el de los electrolitos bajo estudio. Este último aspecto confirma que las dilataciones de ambos materiales durante el proceso de enfriamiento o calentamiento de la pila se ajustan bastante bien no produciéndose en principio, defectos que puedan resultar en el mal funcionamiento de la misma o incluso que puedan conducir a su rotura (12).

Por último, en esta misma tabla se recogen las características de cada uno de los materiales cerámicos utilizados en la elaboración de los distintos pares ánodo/electrolito. En ambas temperaturas de cosinterización el cermet Ni-GDC mantiene una microestructura con una porosidad adecuada para el correcto transporte de los gases ( $25 \%$ porosidad abierta) así como una elevada conductividad eléctrica $\left(\sigma_{700^{\circ} \mathrm{C}}=\sim 4 \times 10^{5}\right.$ $\mathrm{S} / \mathrm{m}$ ) (5). El hecho de que dicho valor (en ambas temperaturas de sinterización) sea un valor cercano a la conductividad del níquel metálico puro a la misma temperatura $\left(\sigma_{700^{\circ} \mathrm{C}}=\sim 21 \times 10^{5}\right.$ $\mathrm{S} / \mathrm{m})(9)$, verifica que existe una buena interconectividad de las partículas metálicas a través de todo el cermet, predominando los contactos Ni-Ni y no produciéndose su engrosamiento, como se puede observar en la figura 5 ( $\mathrm{a}$ y b). En cuanto a los 

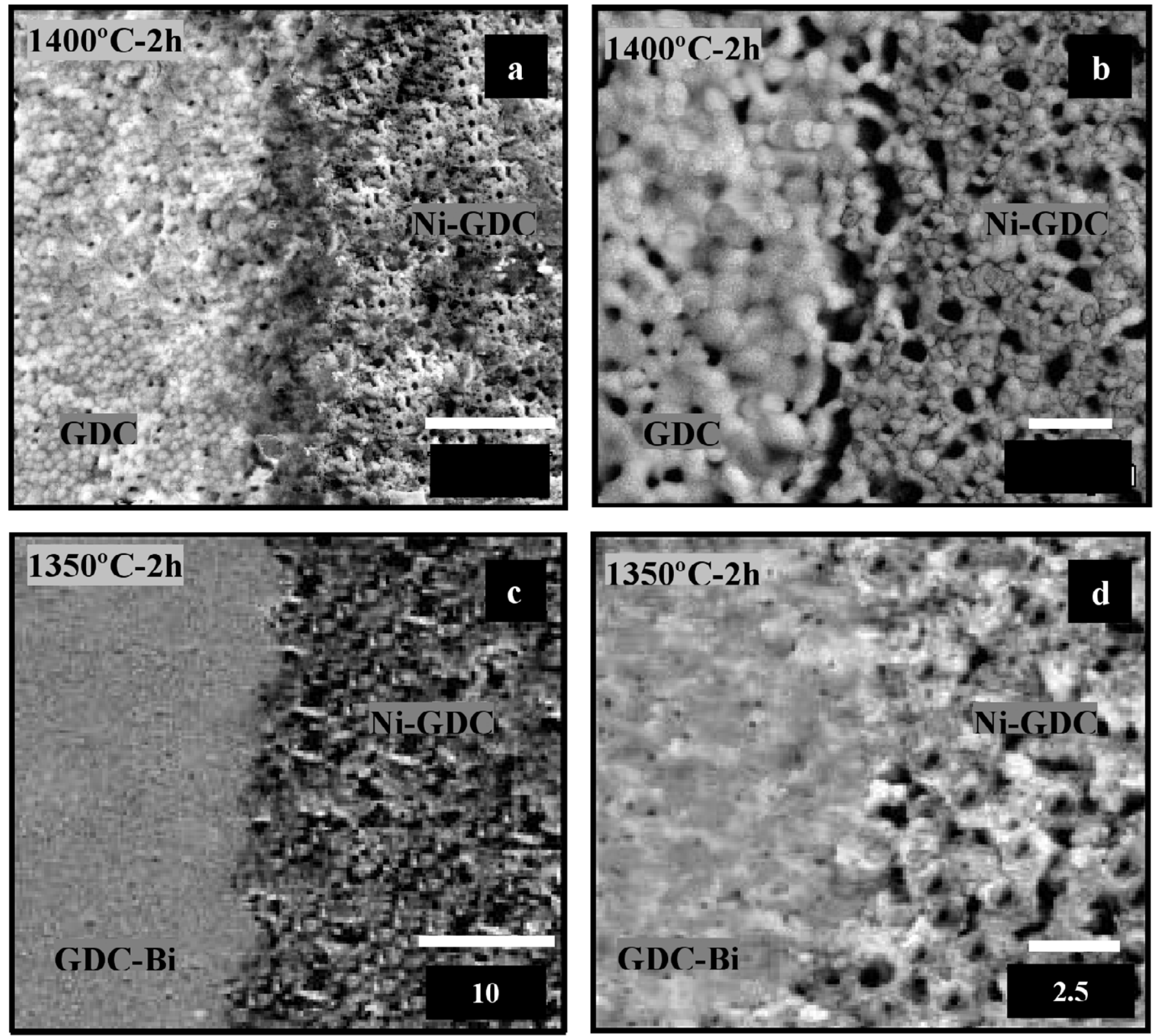

Fig. 3- Micrografías MEB de secciones transversales de sistemas ánodo/electrolito cosinterizados a las temperaturas indicadas, (a,d) superficies de fractura y $(b, c)$ superficies pulidas.

electrolitos, tanto el obtenido por vía química y sinterizado a $1400^{\circ} \mathrm{C}$ durante $2 \mathrm{~h}$ como el modificado con óxido de bismuto y sinterizado a $1350^{\circ} \mathrm{C}$ durante $2 \mathrm{~h}$, presentan unas propiedades eléctricas adecuadas $\left(\sigma_{\text {total }}=3-4 \mathrm{~S} / \mathrm{m}\right.$ a $\left.700^{\circ} \mathrm{C}\right)$ y unos muy

TABLA I. CARACTERÍSTICAS PRINCIPALES DE LOS MATERIALES CERÁMICOS QUE CONSTITUYEN LOS PARES ÁNODO/ELECTROLITO.

\begin{tabular}{|c|c|c|c|c|c|}
\hline \multirow{2}{*}{ Composición } & $\begin{array}{c}\text { Condiciones } \\
\text { de } \\
\text { sinterización }\end{array}$ & $\begin{array}{c}\text { Densidad } \\
\text { relativa }\end{array}$ & $\sigma_{\mathrm{T}}\left(600^{\circ} \mathrm{C}\right)$ & $\sigma_{\mathrm{T}}\left(700^{\circ} \mathrm{C}\right)$ & $\mathrm{CET}$ \\
\cline { 3 - 6 } & $(\mathrm{S} / \mathrm{m})$ & $(\mathrm{S} / \mathrm{m})$ & $\begin{array}{c}\alpha \times 10^{6} \\
\left(\mathrm{~K}^{-1}\right)\end{array}$ \\
\hline \multirow{2}{*}{$\begin{array}{c}\mathrm{Ni} \text {-GDC con } \\
\text { un 39\% en } \\
\text { vol. de Ni* }\end{array}$} & $1400^{\circ} \mathrm{C} 2 \mathrm{~h}$ & $\sim 76$ & $\begin{array}{c}\sim 14.1 \\
\mathrm{x} 10^{5}\end{array}$ & $\begin{array}{c}\sim 14.9 \\
\mathrm{x} 10^{5}\end{array}$ & $\sim 13.5$ \\
\cline { 2 - 6 } & $1350^{\circ} \mathrm{C} 2 \mathrm{~h}$ & $\sim 75$ & $\begin{array}{c}\sim 14.2 \\
\mathrm{x} 10^{5}\end{array}$ & $\begin{array}{c}\sim 14.6 \\
\mathrm{x} 10^{5}\end{array}$ & $\sim 13.5$ \\
\hline $\mathrm{GDC}$ & $1400^{\circ} \mathrm{C} 2 \mathrm{~h}$ & $\sim 95$ & $\sim 0.7$ & $\sim 3.2$ & $\sim 12.7$ \\
\hline $\mathrm{GDC}-\mathrm{Bi}$ & $1350^{\circ} \mathrm{C} 2 \mathrm{~h}$ & $\sim 97$ & $\sim 2.1$ & $\sim 3.9$ & $\sim 12.6$ \\
\hline
\end{tabular}

\footnotetext{
* Cermet obtenido a partir de un composite con un $50 \%$ en peso de $\mathrm{NiO}$.
}

buenos valores de densidad relativa $\left(95-97 \% \mathrm{D}_{\mathrm{t}}\right.$ ), características adecuadas para asegurar el buen funcionamiento de la celda.

\section{CONCLUSIONES}

De acuerdo a las observaciones experimentales realizadas, los cermets de composición Ni-Ce ${ }_{0.9} \mathrm{Gd}_{0.1} \mathrm{O}_{1.95}$ con un $39 \%$ en volúmen de $\mathrm{Ni}$ son compatibles químicamente con los electrolitos basados en ceria dopada con gadolinia $\left(\mathrm{Ce}_{0.9} \mathrm{Gd}_{0.1} \mathrm{O}_{1.95}\right)$, al menos hasta temperaturas tan elevadas como de $1450^{\circ} \mathrm{C}$.

En los pares cosinterizados, no existe la presencia de una cierta migración o interdifusión de ciertas especies a lo largo de la región interfacial, desde el cermet hacia el electrolito o viceversa. Además, las interfases están bien definidas y no presentan grietas o delaminaciones, características óptimas para el correcto funcionamiento de la celda. 


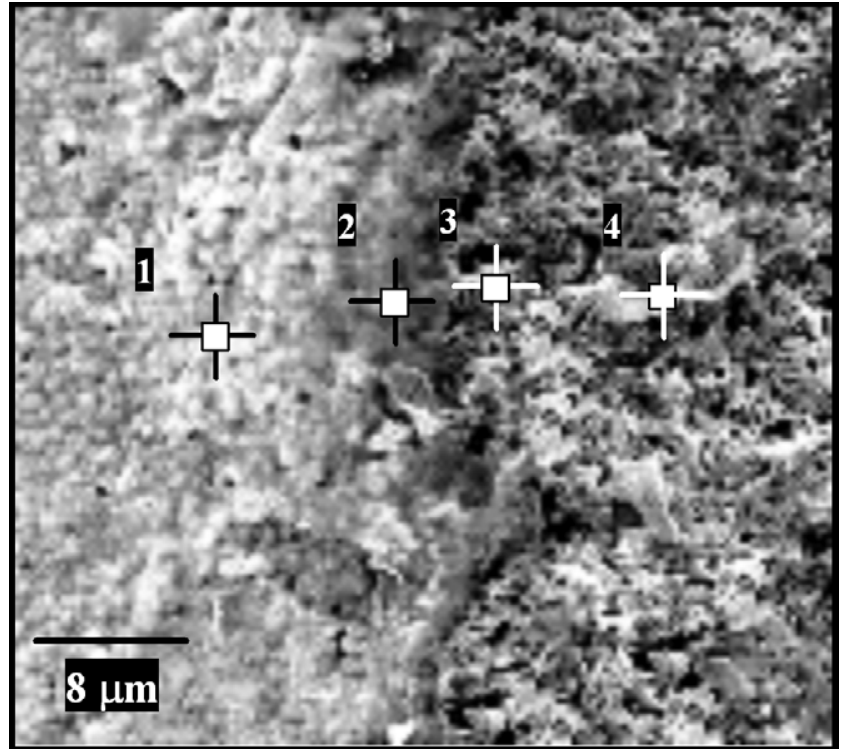

\begin{tabular}{|l|l|l|l|}
\hline Punto & $\% \mathrm{Ni}$ & $\% \mathrm{CeO}_{2}$ & $\% \mathrm{Gd}_{2} \mathrm{O}_{3^{\infty}}$ \\
\hline 1 & ---- & $\sim 95$ & $\sim 5$ \\
\hline 2 & ---- & $\sim 94$ & $\sim 6$ \\
\hline 3 & $\sim 43$ & $\sim 54.5$ & $\sim 2.5$ \\
\hline 4 & $\sim 44$ & $\sim 53.3$ & $\sim 2.7$ \\
\hline
\end{tabular}
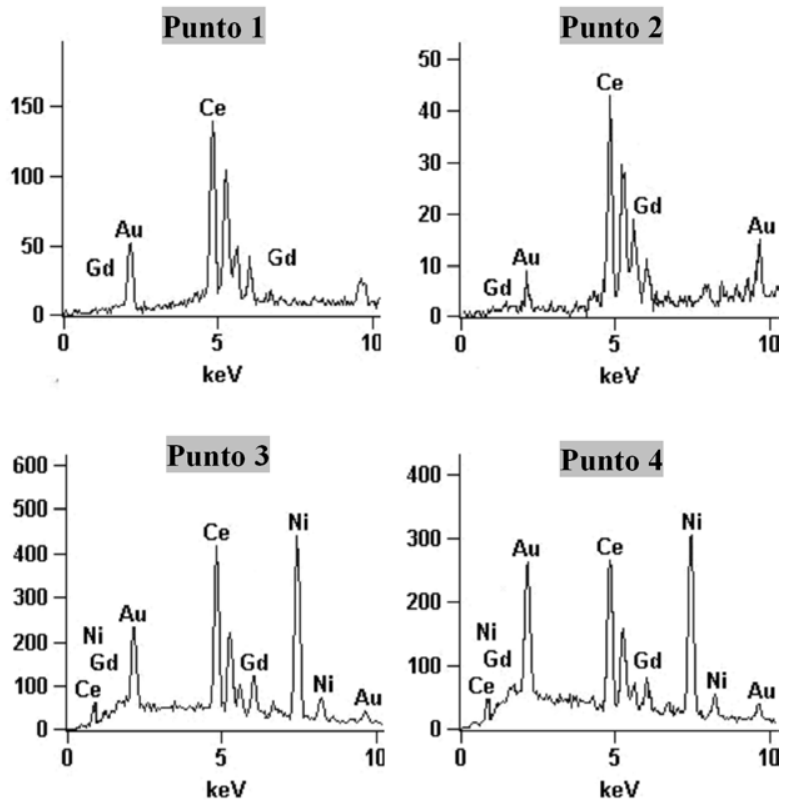

Fig. 4- Micrografía MEB de un sistema Ni-GDC/GDC sinterizado a $1400^{\circ} \mathrm{C}$ durante $2 \mathrm{~h}$ y análisis composicional obtenido por EDAX sobre los puntos marcados a través de la región interfacial (gráficas y tabla).

Los cermets, $\mathrm{Ni}-\mathrm{Ce}_{0.9} \mathrm{Gd}_{0.1} \mathrm{O}_{1.95}$, son compatibles termomecánicamente con las cerámicas $\mathrm{Ce}_{0.9} \mathrm{Gd}_{0.1} \mathrm{O}_{1.95}$, presentando valores de coeficientes de expansión térmica que resultan ser muy próximos entre sí.

La presencia en el electrolito de una pequeña cantidad de óxido de bismuto ( $1.0 \%$ en peso de $\mathrm{Bi}_{2} \mathrm{O}_{3}$ ) como ayudante de sinterización no afecta ni a la compatibilidad química de la cerámica con el cermet ni a su comportamiento termomecánico.
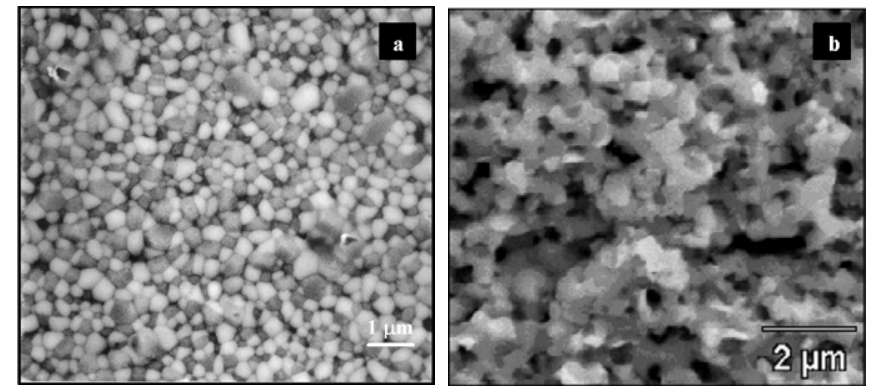

Fig. 5- Micrografías MEB (a) de la superficie pulida de un composite NiO-GDC sinterizado a $1350^{\circ} \mathrm{C}$ durante $2 \mathrm{~h}$ y (b) de la fractura del mismo composite después de reducir, (Ni-GDC).

Por lo tanto se puede concluir que, ambos materiales, NiGDC y GDC ó GDC-Bi se presentan como unos excelentes materiales cerámicos para formar en cosinterización una futura semicelda SOFC de temperatura intermedia.

\section{AGRADECIMIENTOS}

Este trabajo ha sido realizado dentro de un proyecto financiado por la comisión Interministerial de Ciencia y Tecnología de España (CICYT, Proyecto Ref. MAT2003-01163). Los autores también agradecen a la Comunidad Autónoma de Madrid la concesión de una beca predoctoral FPI.

\section{BIBLIOGRAFÍA}

1. D. W. Dees, T.D. Claar, T.E. Easler, D.C. Fee, F.C. Mrazek, Conductivity of Porous $\mathrm{Ni} / \mathrm{ZrO}_{2}-\mathrm{Y}_{2} \mathrm{O}_{3}$ cermets, J. Electrochem. Soc., 134, 9, 2141-2146 (1987).

2. W.Z. Zhu, S.C. Deevi, A review on the status of anode materials for solid oxide fuel cells, Mat. Sci. Eng. A, 362, 1-2, 228-239 (2003).

3. S.J.A. Livermore, J.W. Cotton, R.M. Ormerod, Fuel reforming and electrical performance studies in intermediate temperature ceria-gadolinia based SOFCs, J. Power Sources, 86, 1-2, 411-416 (2000).

4. B.C.H. Steele, Appraisal of $\mathrm{Ce}_{1-\mathrm{Gd}} \mathrm{Gd}_{2}$ electrolytes for IT-SOFC operation at $500^{\circ} \mathrm{C}$, Solid State Ionics, $129,1-4,95-110(2000)$.

5. V. Gil, C. Moure, J. Tartaj, Sinterability, microestructure and electrical properties of $\mathrm{Ni} / \mathrm{Gd}$-doped Ceria cermets used as anode materials for SOFCs, J. Eur. Ceram. Soc., 27, 4205-4209 (2007).

6. V. Gil, Tesis Doctoral: Preparación y caracterización de materials cerámicos para su uso como components: electrolito, ánodo y cátodo, en pilas de combustible de óxido sólido de termperatura intermedia. Estudio de sus compatibilidades, (2006).

7. V. Gil, J. Tartaj, C. Moure, P. Durán, J. Eur. Ceram. Soc., Effect of $\mathrm{Bi}_{2} \mathrm{O}_{3}$ addition on the sintering and microstructural development of gadolinia-doped ceria ceramics, 27, 701-805 (2007).

8. V. Gil, J. Tartaj, C. Moure, P. Durán, Sintering, microstructural development and electrical properties of gadolini-doped ceria electrolyte with bismuth oxide as a sintering aid, J. Eur. Ceram. Soc., 26, 3161-3171 (2006).

9. Gmelins Handbuch der anorganischen Chemie, Ni [A II], p. 355.

10. H. Hayashi, M. Kanoh, C.J. Quan, H. Inaba, S.R. Wang, M. Dokiya, H.Tagawa, Thermal expansion of Gd-doped ceria and reduced ceria, Solid State Ionics, 132, 227-233 (2000)

11. P. Bance, N.P.Brandon, B. Girvan, P. Holbeche, S. O'Çdea, B.C.H. Steele, Spinning-out a fuel cell company from a UK University-2 years of progress at Ceres Power, J. Power Sources, 131, 86-90 (2004).

12. J. Laurencin, B. Morel, Y. Bultel, F. Lefebvre-Joud, Thermo-mechanical model of solid oxide fuel cell fed with methanex, Fuel Cells, 6, 1, 64-70 (2006).

Recibido: 31.07.07

Aceptado: 20.12.07 